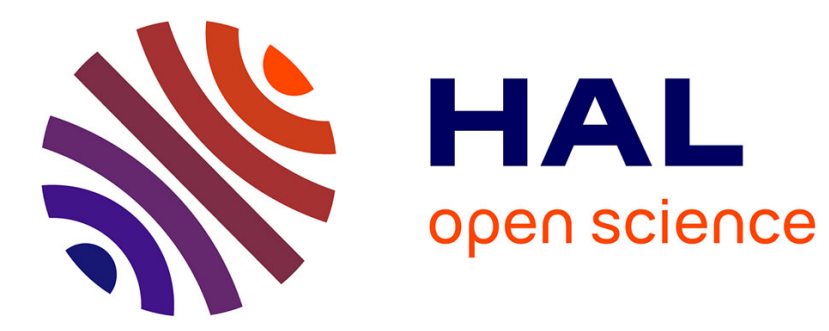

\title{
Control of chaos via OGY method on a bistable energy harvester
}

\author{
Leonardo Rocha de La Roca, João Peterson, Marcelo da Cruz Pereira, \\ Americo Cunha Jr
}

\section{- To cite this version:}

Leonardo Rocha de La Roca, João Peterson, Marcelo da Cruz Pereira, Americo Cunha Jr. Control of chaos via OGY method on a bistable energy harvester. 25th ABCM International Congress on Mechanical Engineering (COBEM 2019), Oct 2019, Uberlândia, Brazil. hal-02388460

\section{HAL Id: hal-02388460 https://hal.science/hal-02388460}

Submitted on 1 Dec 2019

HAL is a multi-disciplinary open access archive for the deposit and dissemination of scientific research documents, whether they are published or not. The documents may come from teaching and research institutions in France or abroad, or from public or private research centers.
L'archive ouverte pluridisciplinaire HAL, est destinée au dépôt et à la diffusion de documents scientifiques de niveau recherche, publiés ou non, émanant des établissements d'enseignement et de recherche français ou étrangers, des laboratoires publics ou privés. 


\section{COBEM2019-1970 \\ CONTROL OF CHAOS VIA OGY METHOD ON A BISTABLE ENERGY HARVESTER}

Leonardo de la Roca

João Victor Ligier Lopes Peterson

Marcelo da Cruz Pereira

Americo Cunha Jr

NUMERICO - Núcleo de Modelagem e Experimentação Computacional

Universidade do Estado do Rio de Janeiro - Rua São Francisco Xavier, 524, Maracanã, Rio de Janeiro - RJ

delaroca@protonmail.com I joao.peterson@uerj.br I marcelo.pereira@uerj.br I americo@ime.uerj.br

Abstract. The current paper aims to use methods of control of chaos to stabilize the chaotic behavior of a nonlinear piezoelectric energy harvester, making its power generation more fruitful, both for signal improvement and for the amplitude of the generated power. We have implemented the "discrete" OGY method of chaos control on the dynamical model of the harvester's system. The methodology (following the OGY method) is based on detecting recurrent points in a Poincaré section, through the development of the dynamical system, and, once the desired orbit is detected (if it exists within the analysis time) the system applies small control forces to maintain the system in this trajectory. Depending on the constraint imposed on the recurring points, stabilization can occur for orbits of not only one period, but also of multiple periods, with different geometries in the phase space, which may take more or less time to stabilize. The results obtained so far are promising. Stabilized orbits generally have a higher energy yield than that of the free system (which may be chaotic or not). Depending on the constraint imposed, the system can be stabilized in larger or smaller orbits of one or multiple periods. In some cases, stabilization may occur for orbits that have an energy efficiency lower than that of the chaotic system, but other orbits, for the same system conditions, achieve a higher yield.

Keywords: Control of Chaos, Energy Harvesting, Nonlinear Oscillator

\section{INTRODUCTION}

The fast pace of technological innovation demands new and more efficient sources of energy to sustain its needs and increase the lifespan of electronic devices. Those demands stimulates the research for the development of more efficient types of energy harvesting (Lopes et al., 2017a,b; Peterson et al., 2018; ?) . Energy harvesting devices are able to assist and preserve the independence and autonomy of many kinds of electronic equipments, from engineering applications of all kinds as in nanotechnology (Koka et al., 2014; Seol et al., 2013; Wang and Song, 2006) and telecommunications to medicine as, for instance, a power source for pacemakers (?).

Vibration energy harvesters are specially useful as vibrations can be generated by various types of sources, from sound, heat, pressure, even a simple human movement can make it capture the exceeding energy (Peterson et al., 2018; Lopes et al., 2019a,b).

Linear energy harvesters can be very effective when vibrating in their natural frequency, more so than nonlinear ones. However, the nonlinearity can provide a wider range of acceptable frequencies (Cottone et al., 2009), and, by doing so, it proves itself being more useful for a greater variety of activities. The problem with nonlinearity is that it encompasses chaotic phenomena in its exercise.

Chaos has, traditionally, been an enemy of engineering. Irregular or completely unexpected results are situations that cannot be afforded in rigorous, precise projects. Even when its presence is unavoidable, it is often an annoyance that brings damage or lessens the efficiency of a given structure. Methods of control of chaos are a good prospects into making chaotic events less harmful or even beneficial to a given system.

For the energy harvester system presented in this research, chaos can be a friend in the sense that it amplifies the incoming vibration amplitude granting a higher energy yield. But it can also be an enemy as the chaotic signal is of poor quality, unpredictable and not suitable for direct use in electronic devices. Because of this, control of chaos techniques (such as the OGY method (Ott et al., 1990)) are used as a solution for the negative effects, stabilizing the chaotic system in an orbit embedded in the chaotic attractor and, as discussed throughout the paper, enabling a predictable, regular and often higher electrical signal. 


\section{BISTABLE ENERGY HARVESTER SYSTEM}

The mechanical system of interest in this work is depicted in Fig. 1. It is the bistable piezoelectric energy harvesting system proposed by Erturk et al. (2009), based on the concept of nonlinear vibrating energy harvesting introduced by Cottone et al. (2009).

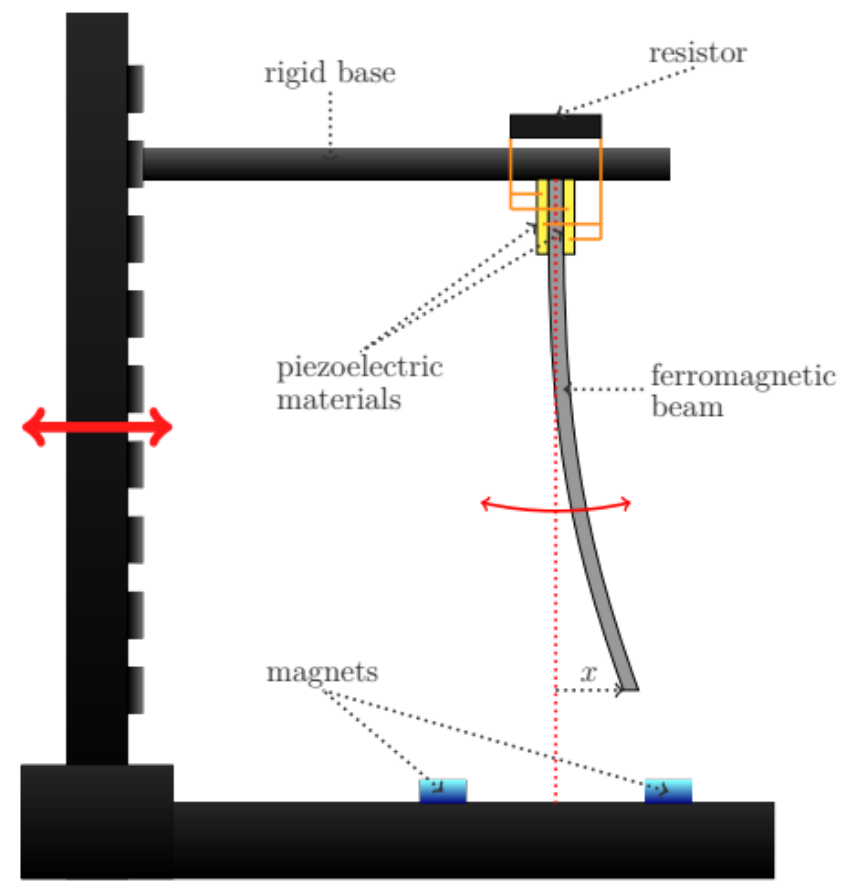

Figure 1: Schematic representation of a nonlinear bistable energy harvester.

The harvester's dynamics is modelled by the following nondimensional equations

$$
\begin{aligned}
& \ddot{x}+2 \xi \dot{x}-\frac{1}{2} x\left(1-x^{2}\right)-\chi v=f \cos \Omega t, \\
& \dot{v}+\lambda v+\kappa \dot{x}=0
\end{aligned}
$$

where $v$ represents the voltage through the resistor, $x$ the horizontal displacement of the beam's tip, $t$ the time, $\chi$ the mechanical coupling factor, $\kappa$ the electric coupling factor, $\lambda$ the inverse characteristic time, $f$ the excitation amplitude and $\Omega$ the excitation frequency. The above equations are supplemented by the initial conditions $x(0)=x_{0}, \dot{x}(0)=\dot{x}_{0}$ and $v(0)=v_{0}$.

For the simulations reported in this work the following values were employed: $\xi=0.01, \kappa=0.5, \chi=0.05, \lambda=0.05$, $\Omega=0.8$, with initial conditions $\left(x_{0}, \dot{x}_{0}, v_{0}\right)=(1,0,0)$.

The pair of magnets act as attractors of the dynamical system, in which the orbits of the phase space revolves around (considering the movement of the vertical beam's free extremity). The system's trajectory can present chaotic behaviour in the range $0.03 \leq f<0.112$ (Barbosa et al., 2015). In order to take advantage of such chaos, seeking to improve the system efficiency, the OGY method (Kapitaniak, 2012; Ott et al., 1990) for control of chaos is used to stabilize the system's dynamics into an unstable periodic orbit embedded in the underlying chaotic attractor.

The power generated and consumed by the system are given, respectively, by

$$
P G_{a v g}=\frac{1}{T} \int_{0}^{T} P G d t
$$

and

$$
P C_{a v g}=\frac{1}{T} \int_{0}^{T} P C d t,
$$


where $P G$ is the power generated, calculated by squaring the instant generated nondimensional voltage $v$ and multiplying it by the inverse characteristic time $\lambda$ (which is inversely proportional to the resistance of the circuit). Therefore $P G=$ $\lambda v^{2}$. And $P C$ is the consumed power, calculated by multiplying the control force (the discrete control force of the OGY method, each cycle) by the velocity of the system at that time of actuation. Therefore $P C=($ ControlForce $) \dot{x}$.

The intensity of the control force is given by the displacement component of the vector " $K$ " multiplying the change in displacement in the Poincaré section from one cycle to the next, better described in the "OGY METHOD" subsection.

The sample period, from "zero to $T$ ", can be any period of time of interest. Here, samples from the moment the control system starts to apply the control force until an arbitrary moment in the permanent state were taken (however, some of the figures only consider the permanent state, to show the stabilized system).

\section{METHODS OF CONTROL OF CHAOS}

Methods of control of chaos are based on stabilizing a given chaotic system through small, wisely decided, disturbances to maintain itself in a certain unstable periodic orbit (UPO).

Although there are several techniques available, most approaches are either based on discrete control methods (such as the OGY method) or continuos control methods (such as the Pyragas method). Both of them require information about the current state of the system (for example, its position and velocity) in order for the method to work properly. As they need information of the outcome of the system to send the control signal, they are categorized as "closed loop" or "feedback" methods (Boccaletti et al., 2000).

The focus of this paper is centered on the OGY method, where most of the results shown here were executed. Figure 2 displays a simplified model of the control system.

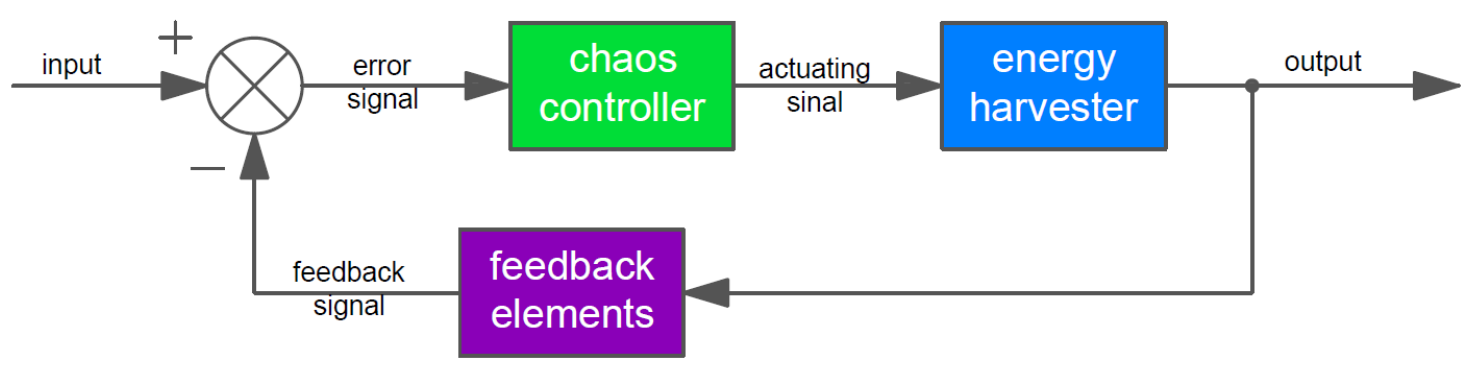

Figure 2: Schematic representation of the chaos controller.

\subsection{OGY METHOD}

Named after E. Ott, C. Grebogi and J. A. Yorke (Ott et al., 1990), this discrete method actuates once (if needed) at each period $\frac{2 \pi}{\Omega}$ of time (a "cycle"), sending a control signal that, through a small controlled force, keeps the system in the chosen unstable periodic orbit (UPO). Such UPO is determined by finding recurrent points in a Poincaré section (points that, after a $\frac{2 \pi}{\Omega}$ period of time, are sufficiently close to each other by an imposed tolerance). While the UPO is being determined, the method cannot actuate, as the desired orbit is not yet defined. This stage of determining the UPO is the "learning stage" of the control system. The system will remain uncontrolled until such condition is met.

In a time-discretized system, a Poincaré map can be given by

$$
\xi_{n+1}=g\left(\xi_{n}, p\right)
$$

where $\xi, p$ and $g$ represent the state of the system, the model parameters and the function capturing the points of the Poincaré map (marking them once per cycle in a displacement vs velocity plane), respectively. If the desired orbit is given by the state $\xi^{*}$ in the Poincaré map and considering that its vicinity is sufficiently small, by an imposed tolerance, we can approximate the deviation of the point $\xi_{n+1}$ in relation to the point $\xi^{*}$ linearly as a function of the deviation of the point $\xi_{n}$ compared to the same point. Following

$$
\left(\xi_{n+1}-\xi^{*}\right)=J\left(\xi_{n}-\xi^{*}\right)+C\left(p-p^{*}\right),
$$

where $J, C$ and $p^{*}$ are, respectively, the Jacobian matrix of the system, the system's derivatives in relation to the control parameter (the excitation force amplitude) and the parameters corresponding to the orbit $Z^{*}$ (Witvoet, 2005). The goal of the method is to make $\left(\xi_{n+1}-\xi^{*}\right)$ decrease, preferably converging to zero. To that end, a gain " $K^{T}$ " is defined such that 


$$
\left(p-p^{*}\right)=-K^{T}\left(\xi_{n}-\xi^{*}\right)
$$

with $K^{T}$ representing the gain vector of the control system. From Eq. (6) and Eq. (7) and denoting the deviations in relation to the target orbit as $\delta$, follows that

$$
\delta \xi_{n+1}=\left(J-C K^{T}\right) \delta \xi_{n}
$$

To make the next deviation decrease in modulus, $J-C K^{T}$ needs to have eigenvalues with a modulus less than 1 . This condition can be solved by the inequality

$$
\left\|K^{T}\right\| \leq \frac{1-\|J\|}{\|C\|}
$$

picking values for $K^{T}$ satisfying it.

In the case of orbits with multiple periods, the recurrent points in the Poincare section must be analysed differently, so the proper unstable periodic orbit may be found. A $n$-periodic orbit is identified when its points in the Poincaré section (denoted by $\xi_{i}$ ) meet the following condition (Neto, 2016):

$$
\left|\xi_{i}-\xi_{i+n}\right|_{i=1}^{N_{p}-n} \leq \text { tol }
$$

where $N_{p}$ is the total number of points in the Poincaré section, tol the imposed tolerance between recurrent points (a small distance, for example), and $n$ the maximum period of the desired orbit. There might be more than one orbit, with different periods, that falls into the same condition. In this case, further restrictions (similar to this one, but with different tolerances) must be implemented to "pinpoint" the desired orbit.

\section{NUMERICAL SIMULATIONS}

Several simulations, for different cases of excitement forces and stabilizations, were done using MATLAB with the OGY method. Apart from the adopted values for the constants $\Omega, \lambda, \xi$ and $\chi$, as previously mentioned, values for $f$ were chosen in the range of $0.03 \leq f<0.112$, as in this range the system presents coexisting attractors, including chaotic responses (Barbosa et al., 2015). The excitation force of $f=0.083$ and $\Omega=0.8$ presents, when uncontrolled, a chaotic behavior. Figures 3, 4 and 5 display some of the effects of the implementation of the OGY method in the system.

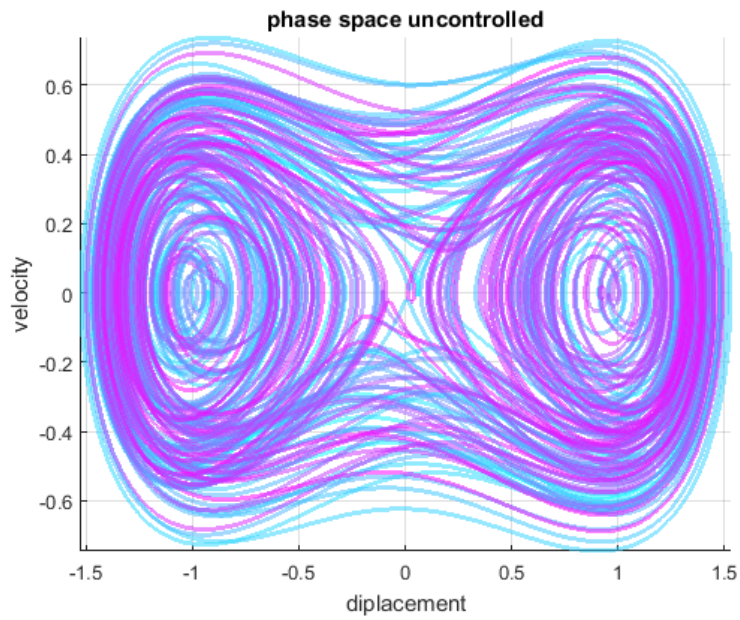

(a) $\mathrm{f}=0.083$ uncontrolled.

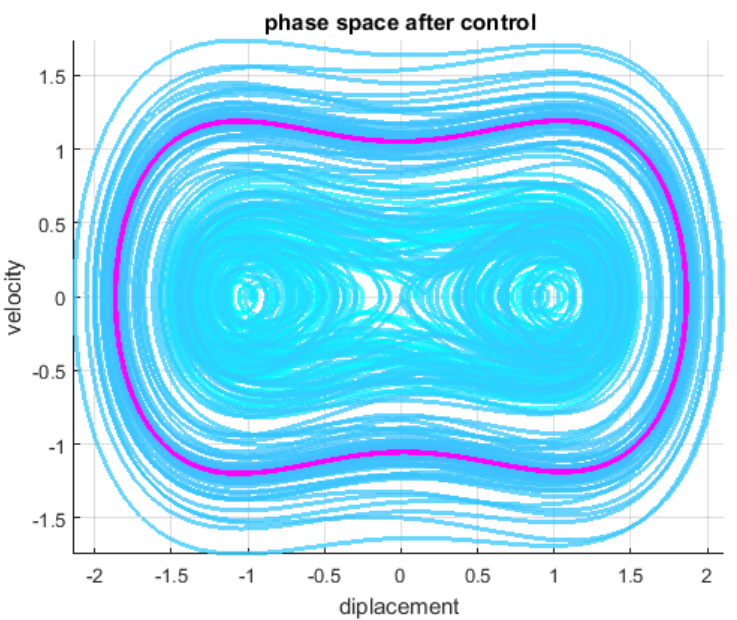

(b) $\mathrm{f}=0.083$ controlled.

Figure 3: Phase space of the case $f=0.083, \Omega=0.8$, uncontrolled and stabilized in a 1-periodic orbit 


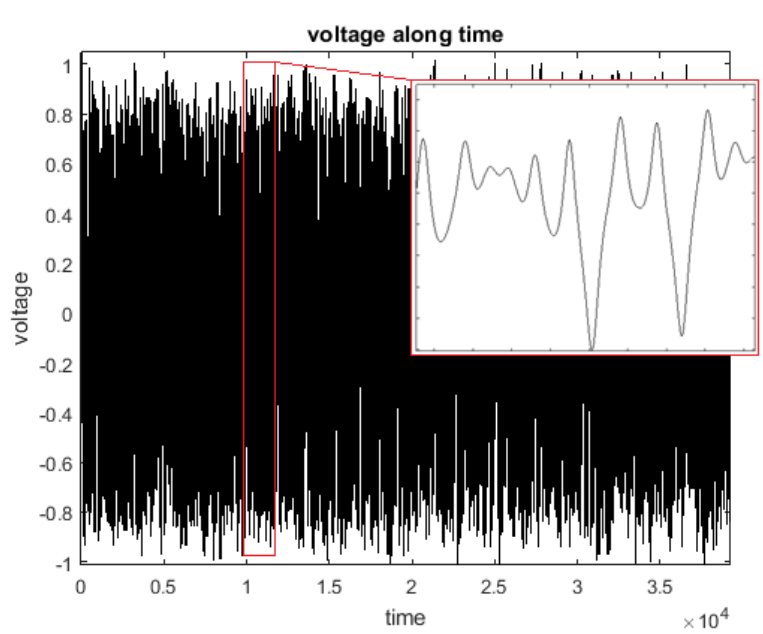

(a) $\mathrm{f}=0.083$ uncontrolled.

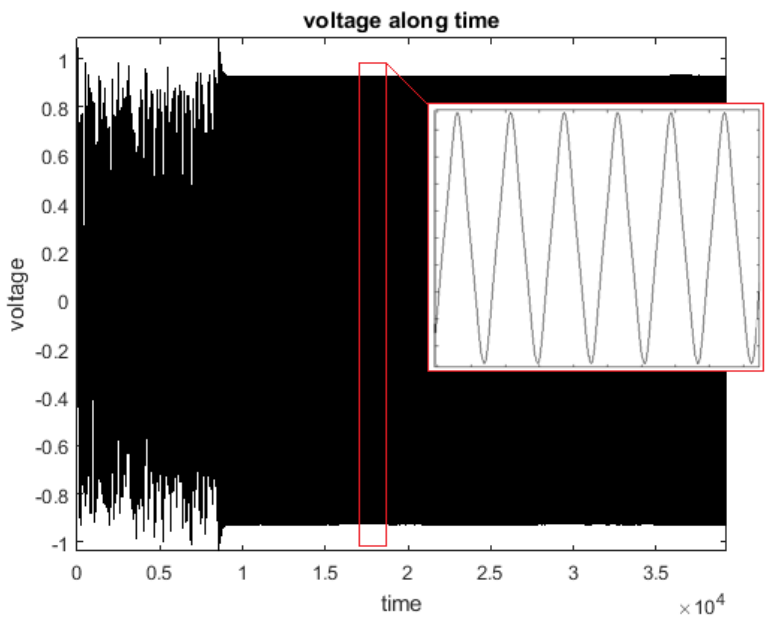

(b) $\mathrm{f}=0.083$ controlled.

Figure 4: Voltage along time for the case $f=0.083, \Omega=0.8$, uncontrolled and stabilized in a 1-periodic orbit

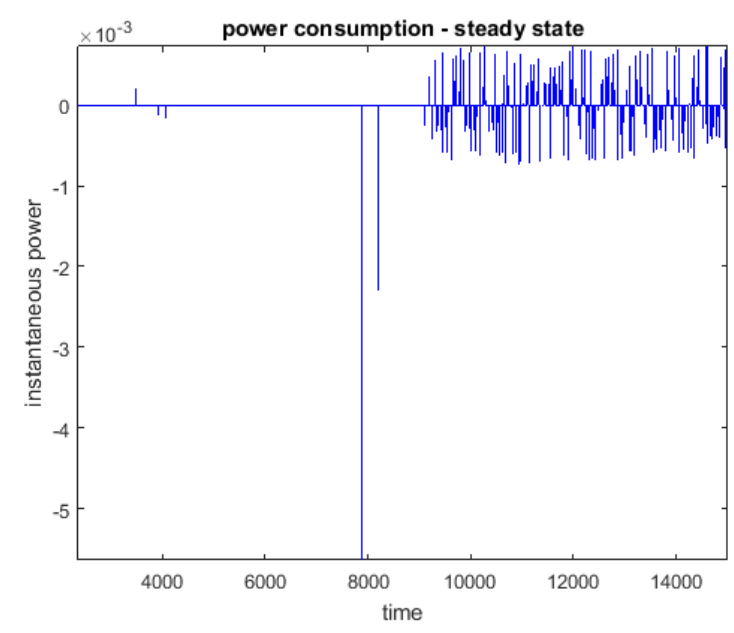

(a) $\mathrm{f}=0.083$ controlled. $P C$ along time.

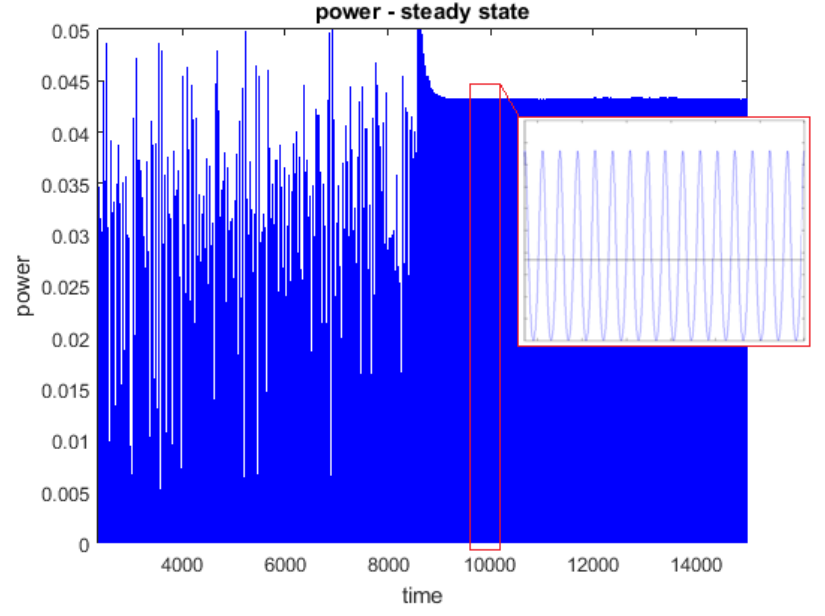

(b) $\mathrm{f}=0.083$ controlled. $P G$ along time.

Figure 5: Power consumption and generation for the case $f=0.083, \Omega=0.8,1$-periodic orbit.

In Figure 3, the colors represent the evolution of the system, from blue to pink, as time progresses. The blue part in Fig. 3(b) shows the transient state before the stabilization in the 1-periodic orbit, in pink.

Figure 4 displays the instant generated voltage along time. The zoom puts in evidence the improvement in the quality of the signal, from a chaotic to a regular, predictable, alternating current.

The last figure of this segment, Fig. 5, indicates the power consumption and generation. Figure 5(a) shows each cycle actuating with discrete impulses from the control force, considering the velocity of the system, defining the power consumption $P C$. Figure 5(b) shows the instant power generation $P G$ along time, directly from the voltage and resistance of the circuit.

Another interesting result is that different orbit stabilizations causes different power outcomes, as shown in Fig. 6, 7, 8 and 9. As it is to be expected, an orbit focused on only one of the attractors (as the case of the 2-periodic orbit in Fig. 8 ) travels an overall shorter distance, making the piezoelectric materials generate less power as result (compared to the larger chaotic orbit of the free, uncontrolled system, in Fig. 6). 


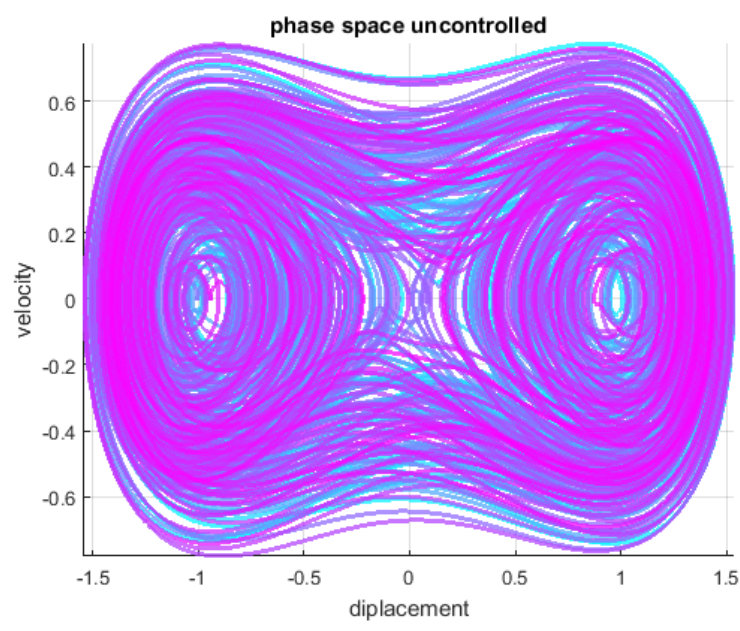

(a) Phase space, $f=0.090$ uncontrolled.

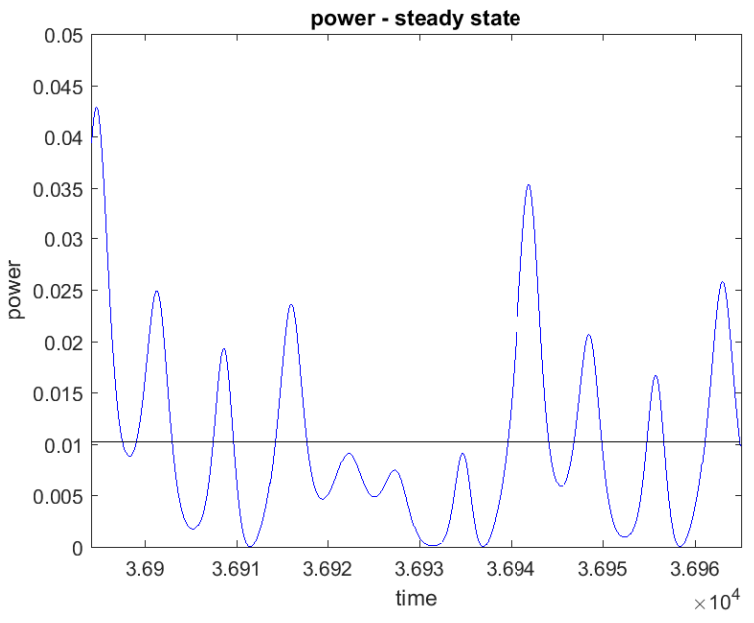

(b) Power generation, $f=0.090$ uncontrolled.

Figure 6: Phase space and power generation for the case $f=0.090, \Omega=0.8$ uncontrolled

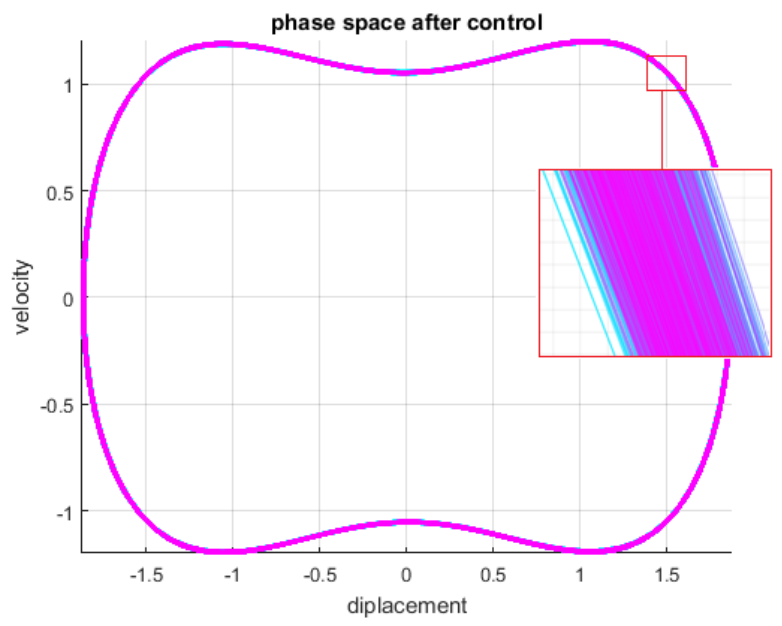

(a) Phase space, $f=0.090$ controlled 1-periodic orbit.

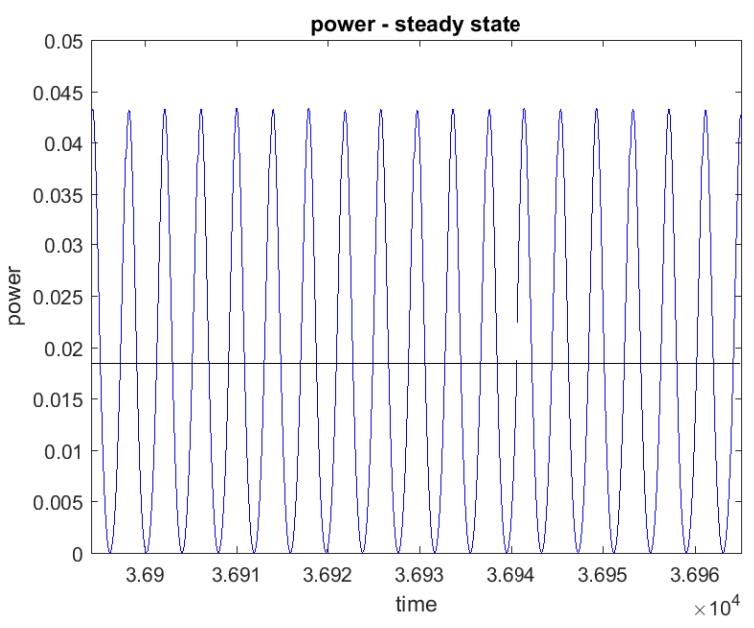

(b) Power generation, $f=0.090$ controlled 1-periodic orbit. Figure 7: Phase space and power generation for the case $f=0.090, \Omega=0.81$-periodic orbit.

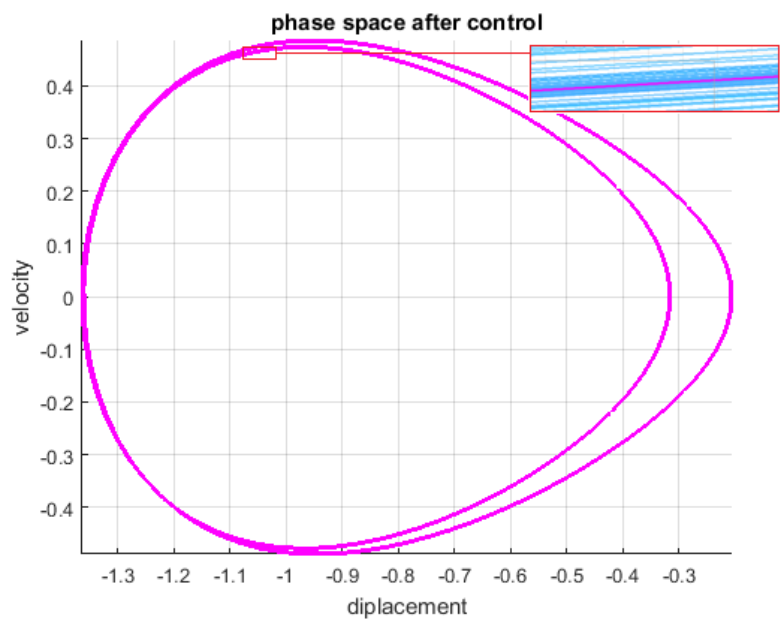

(a) Phase space, $f=0.090$ controlled 2-periodic orbit.

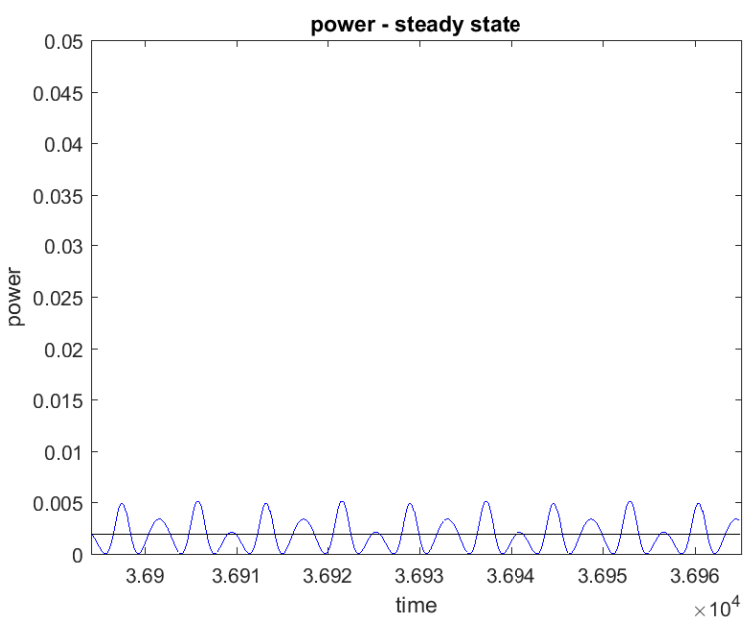

(b) Power generation, $f=0.090$ controlled 2-periodic orbit. Figure 8: Phase space and power generation for the case $f=0.090, \Omega=0.82$-periodic orbit. 


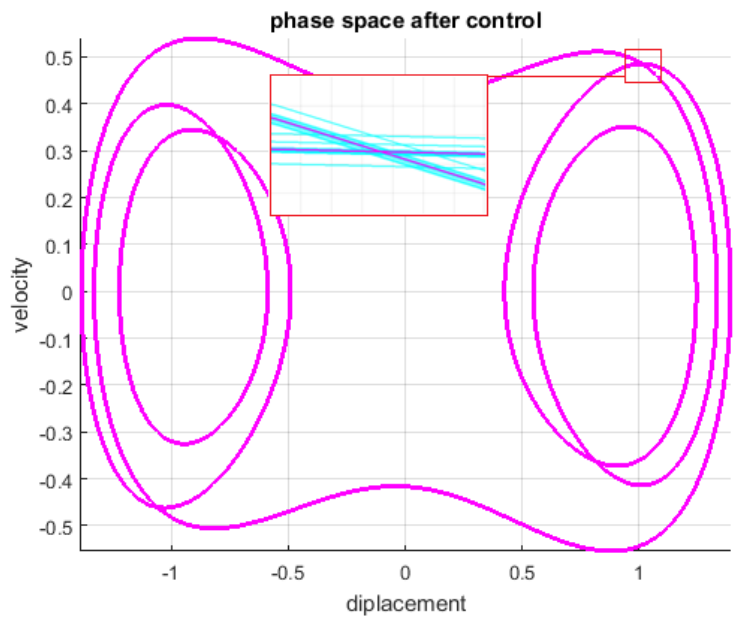

(a) Phase space, $f=0.090$ controlled 5-periodic orbit.

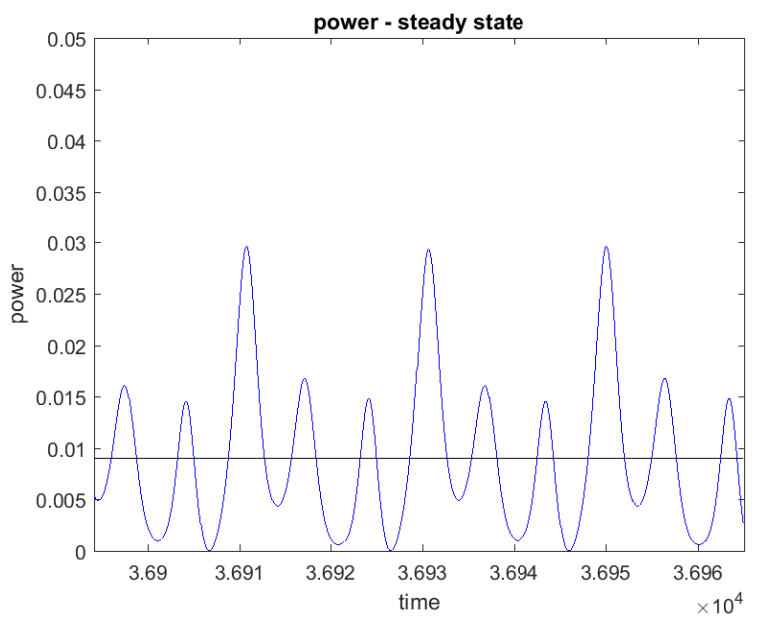

(b) Power generation, $f=0.090$ controlled 5-periodic orbit.

Figure 9: Phase space and power generation for the case $f=0.090, \Omega=0.8$ 5-periodic orbit.

As shown in the graphics, not all cases show improvements. Comparing the power generation figures of the controlled systems (Fig. 7(b), 8(b) and 9(b)) with the uncontrolled one (Fig. 6(b)), it is possible to visualize that the 2-periodic orbit case had a massive decrease in power generation. As for the 1-periodic case, in Fig. 7, it increases, and for the 5-periodic orbit the power generation does not suffer much of a change.

The following table, Table 1, summarizes several results in terms of power generation from the controlled systems in relation to the uncontrolled one.

\begin{tabular}{|c|c|c|c|c|r|}
\hline$f$ & uncontrolled & controlled & controller $\left(\times 10^{-4}\right)$ & effective & enhancement \\
\hline 0.050 & 0.0001 & 0.0068 & -0.0005 & 0.0068 & $68 \times$ \\
\hline 0.083 & 0.0073 & 0.0131 & -0.0006 & 0.0131 & $1.8 \times$ \\
\hline $0.0901-\mathrm{p}$ & 0.0077 & 0.0154 & -0.0001 & 0.0154 & $2 \times$ \\
\hline $0.0902-\mathrm{p}$ & 0.0077 & 0.0037 & -0.0002 & 0.0037 & $0.5 \times$ \\
\hline $0.0905-\mathrm{p}$ & 0.0077 & 0.0084 & -0.0001 & 0.0084 & $1.1 \times$ \\
\hline
\end{tabular}

Table 1: Power generation of the uncontrolled system compared to the controlled one over different values of $f$, with different periodic orbits for the case $f=0.090$ (orbits "1-p"=1-periodic, "2-p"=2-periodic and "5-p"=5-periodic).

The Table 1 indicates the values of the average power generated, $P G_{a v g}$ (displayed in the table as "uncontrolled" and "controlled" for the respective states of the system), as well as the power consumption of the controller, $P C_{a v g}$ (displayed in the table as "controller"). The effective power of the system, given by $P G_{a v g}-P C_{a v g}$ for the controlled cases, is shown in the "effective" column. And at the last column displays the overall improvement of the controlled system compared to its uncontrolled state (the value of 1 would represent no improvement at all, and values below it represent a decay in power generation). The time sample of the simulations were from the moment the control system starts to send signals until an arbitrary moment in the permanent state, with the same time limits for the uncontrolled and controlled systems.

The great improvement of the $f=0.050$ case is mainly due to the initial position of the system, $x_{0}=1$, which, for the uncontrolled state, "trapped" the extremity of the ferromagnetic beam around one of the magnets, as the initial displacement was near it. The control system was able to break free from this "trap" as the control forces set the orbit around both magnets, greatly extending the travelled distance, thus increasing the power generated by the piezoelectric components.

It can also be seen the decay in efficiency for the case " $f=0.090$ 2-p", considering Fig. 8 in relation to Fig. 6 . The increase of almost two fold the power generated for the case " $f=0.0901-p "$ and the roughly indifferent case " $f=0.090$ $1-\mathrm{p} "$, as the average generated power is only about $10 \%$ above the uncontrolled system's power.

\section{CONCLUSION}

This work has the purpose to analyse the usage of control of chaos techniques, in particular the discrete OGY method, as a tool to increase the efficacy of a nonlinear piezoelectric energy harvester. Such improvement comes in the form of a better, regular, electrical signal and an increase in the amplitude of vibration, generating more energy. 
The study and results show that such improvement does not happen for every controlled state, as for certain cases the controlled system decays in power - a "bad effect", and in other situations the control gain might be indifferent in comparison to the uncontrolled system, displaying virtually the same power generation - an "ugly effect". Nonetheless, the correct UPO stabilization may prove to be highly effective for the system's power generation - a "good effect". Different situations can have distinct benefits from the application of control of chaos. As can be seen in Table 1, the case $f=0.050$ shows a drastic increase in power generation while for the cases $f=0.083$ and $f=0.090$ the control "only" offers around two times the uncontrolled generated power.

\section{ACKNOWLEDGEMENTS}

The authors acknowledge the support given to this research by the funding agencies Conselho Nacional de Desenvolvimento Científico e Tecnológico (CNPq), Coordenação de Aperfeiçoamento de Pessoal de Nível Superior - Brasil (CAPES) - Finance Code 001 and Carlos Chagas Filho Research Foundation of Rio de Janeiro State (FAPERJ) under grants E-26/010.002.178/2015, E-26/010.000.805/2018 and E-26/202.983/2018.

\section{REFERENCES}

Barbosa, W., De Paula, A., Savi, M. and Inman, D., 2015. "Chaos control applied to piezoelectric vibration-based energy harvesting systems”. The European Physical Journal Special Topics, Vol. 224, No. 14-15, pp. 2787-2801.

Boccaletti, S., Grebogi, C., Lai, Y.C., Mancini, H. and Maza, D., 2000. "The control of chaos: theory and applications". Physics reports, Vol. 329, No. 3, pp. 103-197.

Cottone, F., Vocca, H. and Gammaitoni, L., 2009. "Nonlinear energy harvesting”. Physical Review Letters, Vol. 102, p. 080601 .

Erturk, A., Hoffmann, J. and Inman, D.J., 2009. "A piezomagnetoelastic structure for broadband vibration energy harvesting". Applied Physics Letters, Vol. 94, p. 254102.

Kapitaniak, T., 2012. Chaos for engineers: theory, applications, and control. Springer Science \& Business Media.

Koka, A., Zhou, Z., Tang, H. and Sodano, H.A., 2014. "Controlled synthesis of ultra-long vertically aligned batio3 nanowire arrays for sensing and energy harvesting applications”. Nanotechnology, Vol. 25, No. 37, p. 375603.

Lopes, V.G., Peterson, J.V.L.L. and Cunha Jr, A., 2017a. "Numerical study of parameters influence over the dynamics of a piezo-magneto-elastic energy harvesting device". In XXXVII Congresso Nacional de Matemática Aplicada e Computacional. São José dos Campos, Brazil.

Lopes, V.G., Peterson, J.V.L.L. and Cunha Jr, A., 2017b. “On the nonlinear dynamics of a bi-stable piezoelectric energy harvesting device". In 24th ABCM International Congress of Mechanical Engineering (COBEM 2017). Curitiba, Brazil.

Lopes, V.G., Peterson, J.V.L.L. and Cunha Jr, A., 2019a. "Analysis of the nonlinear dynamics of a bistable energy harvesting system with colored noise disturbances". Conference of Computational Interdisciplinary Science (CCIS 2019).

Lopes, V.G., Peterson, J.V.L.L. and Cunha Jr, A., 2019b. "Exploring the nonlinear dynamics of bistable energy harvester". XVIII International Symposium on Dynamic Problems of Mechanics (Diname 2019).

Neto, C.C., 2016. "Control of chaos utilizing the extended delayed states feedback method applied on a nonlinear pendulum". Ph.D. thesis, Universidade Federal do Rio de Janeiro. (In Portuguese).

Ott, E., Grebogi, C. and Yorke, J.A., 1990. "Controlling chaos”. Physical Review Letters, Vol. 64, pp. $2837-2837$.

Peterson, J.V.L.L., Lopes, V.G. and Cunha Jr, A., 2016. "Maximization of the electrical power generated by a piezomagneto-elastic energy harvesting device”. In XXXVI Congresso Nacional de Matemática Aplicada e Computacional. Gramado, Brazil.

Peterson, J.V.L.L., Lopes, V.G. and Cunha Jr, A., 2018. "Dynamic analysis and characterization of a nonlinear bi-stable piezo-magneto-elastic energy harvester". MATEC Web of Conferences, Vol. 241, p. 01001. doi: 10.1051/matecconf/201824101001. URL https://doi .org/10.1051/matecconf/201824101001.

Priya, S. and (Editors), D.J.I., 2009. Energy Harvesting Technologies. Springer.

Seol, M.L., Choi, J.M., Kim, J.Y., Ahn, J.H., Moon, D.I. and Choi, Y.K., 2013. "Piezoelectric nanogenerator with a nanoforest structure". Nano Energy, Vol. 2, No. 6, pp. 1142-1148.

Wang, Z.L. and Song, J., 2006. "Piezoelectric nanogenerators based on zinc oxide nanowire arrays". Science, Vol. 312 , No. 5771, pp. 242-246.

Witvoet, G., 2005. "Control of chaotic dynamical systems using ogy". Traineeship Report, Eindhoven University of Technology.

\section{RESPONSIBILITY NOTICE}

The authors are the only responsible for the printed material included in this paper. 